-Supporting Information for-

\title{
Low temperature Heck reactions of axially chiral $o$-iodoacrylanilides occur with chirality transfer: Implications for catalytic asymmetric Heck reactions
}

\author{
Andre J. B. Lapierre, Steven J. Geib and Dennis P. Curran \\ Department of Chemistry, University of Pittsburgh, Pittsburgh, PA 15260 USA \\ E-mail curran@pitt.edu
}

Table of Contents

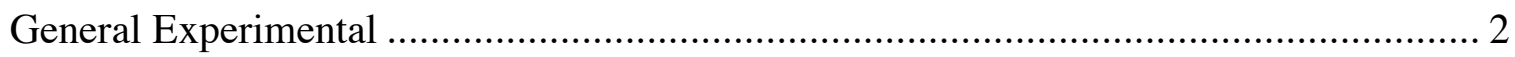

Compound Synthesis and Characterization......................................................... 3

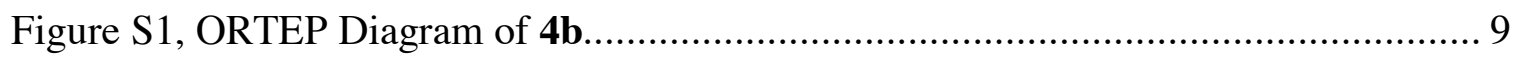

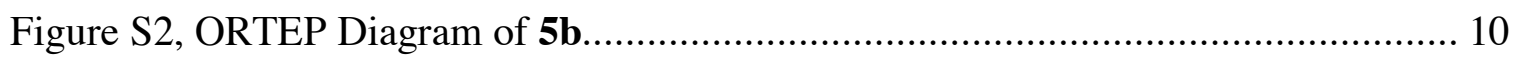

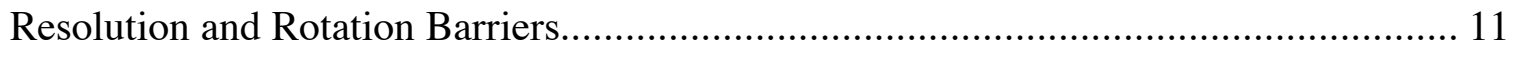


General Experimental: Unless otherwise noted, all reactions were run under an argon atmosphere. All reactions were performed in oven-dried glassware that was cooled under argon. 4-Bromo-2-methylaniline, 2,4-dimethylaniline, benzyltrimethylammonium dichloroiodate, sodium hydride ( $95 \%$ dispersion in oil), methyl iodide, silver sulfate, trimethylaluminum (2.0 M in hexanes), and tris(dibenzylideneacetone)-dipalladium(0)-chloroform adduct were purchased from Aldrich and used without modification, save for the following exception. 2,4Dimethylaniline was dissolved in diethyl ether, filtered through a pad of silica gel to remove colored baseline impurities, and concentrated in vacuo. Ethanol, iodine, and triethylamine were purchased from Fisher Scientific and used without modification. $\operatorname{Pd}(\mathrm{OAc})_{2}$ and tertbutylphosphonium tetrafluoroborate were purchased from Strem Chemicals and used without modification. 2-Iodo-4,6-dimethylaniline 59 was prepared according to Kajigaesi's procedure. ${ }^{1}$ Methyl 2-methylbut-2E-enoate was prepared according to a previously reported procedure. ${ }^{2}$ Air and moisture sensitive chemicals were handled using standard syringe techniques. THF and toluene were dried by passing them through columns of activated alumina.

${ }^{1} \mathrm{H}$ and ${ }^{13} \mathrm{C}$ NMR spectra were recorded on Bruker Avance DPX 300 (300 MHz), Avance 300 (300 MHz), and Avance DRX 500 (500 MHz) NMR spectrometers. Chemical shifts are reported in ppm relative to TMS using the residual solvent proton resonance of $\mathrm{CDCl}_{3}(7.27 \mathrm{ppm})$ or the $\mathrm{CDCl}_{3}$ carbon peak $(77.0 \mathrm{ppm})$. In reporting data, the following abbreviations were employed: $\mathrm{s}$ $=$ singlet, $\mathrm{bs}=$ broad singlet, $\mathrm{d}=$ doublet, $\mathrm{dd}=$ doublet of a doublet, $\mathrm{dq}=$ doublet of a quartet, $\mathrm{qd}$ $=$ quartet of a doublet, $\mathrm{qq}=$ quartet of a quartet, and qn $=$ quintet. IR spectra were recorded as t,hin films or neat on $\mathrm{NaCl}$ plates, on an ATI Mattson Genesis Series on FT-IR spectrometer. Low and high resolution mass spectra were obtained on a Micromass Inc., Autospec with an E-

\footnotetext{
${ }^{1}$ Kajigaeshi, S.; Kakinami, T.; Yamasaki, H.; Fujisaki, S.; Okamoto, T. Bull. Chem. Soc. Jpn. 1988, 61, 600 .

${ }^{2}$ Guldbrandt, M.; Johansen, T. N.; Frydenvang, K.; Bräuner-Osborne, H.; Stensbol, T. B.; Nielsen, B.; Karla, R.; Santi, F.; Krogsgaard-Larsen, P.; Madsen, U. Chirality 2002, 14, 351363.
} 
B-E geometry. Analytical chiral HPLC analyses were conducted using a $(S, S)$-Whelk-O 1 column (Pirkle) and a Waters model $440 \mathrm{UV}$ detector at wavelength $254 \mathrm{~nm}$. Gas chromatograms (GC) were run on an Agilent 19091Z-413E gas chromatograph equipped with an enhanced HPCHEM integrator. An HP-1 capillary methyl siloxane column of $30 \mathrm{~m}$ in length and $0.32 \mathrm{~mm}$ in diameter was used for all runs. The initial temperature of the program was 50 ${ }^{\circ} \mathrm{C}$ with a temperature ramp of $10{ }^{\circ} \mathrm{C} / \mathrm{min}$ up to $315^{\circ} \mathrm{C}$, where after the same temperature is maintained for 10 min further. Thin layer chromatography (TLC) was performed on silica gel 60 F254 glass-backed plates with a layer thickness of $0.25 \mathrm{~mm}$ manufactured by E. Merck. TLC visualization was achieved by illumination with a $254 \mathrm{~nm}$ UV. Flash chromatography was performed silica gel (230-400 mesh ASTM) purchased from Bodman or Sorbtech. Optical rotations were measured on a Perkin-Elmer 241 polarimeter at the Na D-line $(\lambda=589 \mathrm{~nm})$ using a $1 \mathrm{dm}$ cell.<smiles>Cc1cc(Br)cc(I)c1N</smiles>

\section{4-Bromo-2-iodo-6-methylaniline:}

In a round bottom flask equipped with a stir bar was dissolved 4-bromo-2-methyl aniline (1.86 g, $10 \mathrm{mmol})$ in ethanol $(33 \mathrm{~mL})$. In succession, iodine $(2.54 \mathrm{~g}, 10.0 \mathrm{mmol})$ and $\mathrm{Ag}_{2} \mathrm{SO}_{4}(3.11 \mathrm{~g}$, $10.0 \mathrm{mmol}$ ) were added to the reaction mixture. ${ }^{3}$ After stirring for $3 \mathrm{~h}$ the solids were removed by filtration and the resulting solution was concentrated in vacuo. The crude material was redissolved in dichloromethane $(30 \mathrm{~mL})$ and washed with $5 \% \mathrm{NaOH}(25 \mathrm{~mL})$ and water $(25$ $\mathrm{mL}$ ). The organic fraction was dried over $\mathrm{MgSO}_{4}$ and concentrated in vacuo. The resulting crude was purified by silica gel chromatography (hexanes/diethyl ether, 95/5) to give a black, crystalline solid (mp 55-57 ${ }^{\circ} \mathrm{C}$ ) in $67 \%$ yield: IR (thin film, $\mathrm{CH}_{2} \mathrm{Cl}_{2}, \mathrm{NaCl}, \mathrm{cm}^{-1}$ ) 2929, 2851,

\footnotetext{
${ }^{3}$ Sy, W.-W. Synth. Comm. 1992, 22, 3215-3219.
} 
1622, 1463; ${ }^{1} \mathrm{H}$ NMR (300 MHz, $\left.\mathrm{CDCl}_{3}\right) \delta 2.20$ (s, $3 \mathrm{H}$ ), 4.10 (bs, $2 \mathrm{H}$ ), 7.15 (dd, $J=2.2,0.7$ $\mathrm{Hz}, 1 \mathrm{H}), 7.63(\mathrm{dd}, J=2.2,0.5 \mathrm{~Hz}, 1 \mathrm{H}) ;{ }^{13} \mathrm{C} \mathrm{NMR}\left(75 \mathrm{MHz}, \mathrm{CDCl}_{3}\right) \delta$ 18.8, 84.3, 109.8, 128.9, 133.0, 138.2, 144.1; HRMS (EI) calcd for $\mathrm{C}_{7} \mathrm{H}_{7} \mathrm{IBrN} 310.8807$, found 310.8808; LRMS (EI) $\mathrm{m} / \mathrm{z}$ $311\left(\mathrm{M}^{+}, 100\right), 232(11), 184(21), 104$ (31), 76 (27).

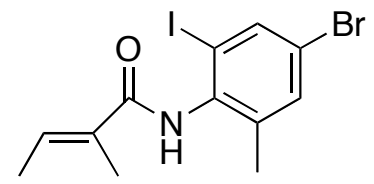

N-(4-Bromo-2-iodo-6-methylphenyl)-2-methyl-(2E)-butenamide:

A dry tube equipped with a stir bar, sealed, and backfilled with Ar. Benzene $(5 \mathrm{~mL})$ was added and the flask cooled to $0{ }^{\circ} \mathrm{C}$. A $2.0 \mathrm{M}$ solution of $\mathrm{AlMe}_{3}(3.75 \mathrm{~mL}, 7.5 \mathrm{mmol})$ was slowly added, ${ }^{4}$ followed by 4-bromo-2-iodoaniline $(1.56 \mathrm{~g}, 5.0 \mathrm{mmol})$ dissolved in benzene $(15 \mathrm{~mL})$. The mixture was allowed to warm to room temperature over $1 \mathrm{hr}$ before methyl 2-methylbut-2Eenoate $(1.14 \mathrm{~g}, 10.0 \mathrm{mmol})$ was added as a solution in benzene $(3.75 \mathrm{~mL})$. The tube was then sealed and then refluxed at $80{ }^{\circ} \mathrm{C}$ for $6 \mathrm{hr}$. When the mixture had cooled to room temperature 1 M HCl $(15 \mathrm{~mL})$ was carefully added, followed by stirring for $1 \mathrm{hr}$. The organic layer was separated and the remaining aqueous layer was extracted with ethyl acetate $(2 \times 15 \mathrm{~mL})$. The combined organic layers were washed with brine $(15 \mathrm{~mL})$, dried with $\mathrm{MgSO}_{4}$, filtered, and concentrated in vacuo. The crude was purified by silica gel flash chromatography (hexanes/diethyl ether, $8 / 2$ ) to give a white, crystalline solid (mp $142-143{ }^{\circ} \mathrm{C}$ ) in $90 \%$ yield: IR (thin film, $\left.\mathrm{CH}_{2} \mathrm{Cl}_{2}, \mathrm{NaCl}, \mathrm{cm}^{-1}\right)$ 2922, 2853, 1663, 1631, 1494; ${ }^{1} \mathrm{H}$ NMR $\left(300 \mathrm{MHz}, \mathrm{CDCl}_{3}\right) \delta$ $1.86(\mathrm{dd}, J=6.9,0.8 \mathrm{~Hz}, 3 \mathrm{H}), 2.01(\mathrm{~s}, 3 \mathrm{H}), 2.28(\mathrm{~s}, 3 \mathrm{H}), 6.71$ (qd, $J=6.9,0.8 \mathrm{~Hz}, 1 \mathrm{H}), 7.04$ (bs, $1 \mathrm{H}), 7.39(\mathrm{~d}, J=1.9 \mathrm{~Hz}, 1 \mathrm{H}), 7.38(\mathrm{~d}, J=1.9 \mathrm{~Hz}, 1 \mathrm{H}) ;{ }^{13} \mathrm{C} \mathrm{NMR}\left(75 \mathrm{MHz}, \mathrm{CDCl}_{3}\right) \delta 12.6$, 14.2, 19.6, 99.7, 121.1, 131.5, 132.6, 133.7, 136.7, 138.4, 139.2, 167.2; HRMS (EI) calcd for $\mathrm{C}_{12} \mathrm{H}_{13} \mathrm{IBrNO}$ 392.9225, found 392.9228; LRMS (EI) $\mathrm{m} / \mathrm{z} 393$ (M+1 47), 311 (37), 268 (81), 83 (92), 55 (100).

\footnotetext{
${ }^{4}$ Artman III, G. D.; Weinreib, S. M. Org. Lett. 2003, 5, 1523-1526.
} 


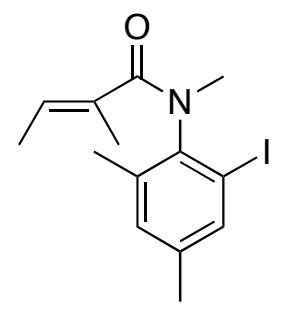

$N$-(2-Iodo-4,6-dimethylphenyl)- $N$,2-dimethyl-(2E)-butenamide (4a):

To a round bottom flask equipped with a stir bar, a slurry of $\mathrm{NaH}(95 \%, 55 \mathrm{mg}, 2.19 \mathrm{mmol})$ and THF ( $1 \mathrm{~mL})$ was created. The flask was capped, backfilled with argon, and its contents cooled to $0{ }^{\circ} \mathrm{C}$. The amide $(600 \mathrm{mg}, 1.82 \mathrm{mmol})$ was dissolved in THF $(5 \mathrm{~mL})$ and added dropwise via syringe to the slurry. After stirring for $1 \mathrm{~h}$ methyl iodide $(127 \mu \mathrm{L}, 2.19 \mathrm{mmol})$ was added via syringe. After a further $15 \mathrm{~min}$ water was carefully added to quench remaining hydride. The resulting biphasic mixture was extracted with diethyl ether $(3 \times 5 \mathrm{~mL})$. The combined organic fractions were washed with brine, dried over $\mathrm{MgSO}_{4}$, and concentrated in vacuo. The resulting crude material was purified by silica gel chromatography (hexanes/diethyl ether, 7/3) to give a white, crystalline solid ( $\mathrm{mp} 80-82{ }^{\circ} \mathrm{C}$ ) in $77 \%$ yield: IR (thin film, $\mathrm{CH}_{2} \mathrm{Cl}_{2}, \mathrm{NaCl}, \mathrm{cm}^{-1}$ ) 2921 , 1634, 1472, 1354; Note: Compound exists as a 3.2:1 mixture of $\mathrm{E} / \mathrm{Z}$ amide rotamers in $\mathrm{CDCl}_{3}$. ${ }^{1} \mathrm{H}$ NMR $\left(300 \mathrm{MHz}, \mathrm{CDCl}_{3}\right) \delta 1.48(\mathrm{~d}, \mathrm{~J}=6.8 \mathrm{~Hz}, 3 \mathrm{H}), 1.65$ (s, $\left.3 \mathrm{H}\right), 2.22(\mathrm{~s}, 3 \mathrm{H}), 2.27(\mathrm{~s}, 3$ H), $3.15(\mathrm{~s}, 3 \mathrm{H}), 5.79(\mathrm{qd}, J=6.8,1.1 \mathrm{~Hz}, 1 \mathrm{H}), 7.00(\mathrm{~s}, 1 \mathrm{H}), 7.52(\mathrm{~s}, 1 \mathrm{H}) ; 13 \mathrm{C}$ NMR $(75$ $\left.\mathrm{MHz}, \mathrm{CDCl}_{3}\right) \delta 13.5,13.8,19.1,20.3,35.9,100.5,130.0,132.0,132.0,136.9,137.9,138.2$, 139.1, 172.7; HRMS (EI) calcd for $\mathrm{C}_{14} \mathrm{H}_{18}$ INO 344.0511, found 344.0503; LRMS (EI) $\mathrm{m} / z 344$ $\left(\mathrm{M}+\mathrm{H}^{+}, 52\right), 216(100), 83$ (78). The racemate was submitted to preparative chiral HPLC separation (Regis Technologies $(S, S)-$ Whelk-O 1; $25 \mathrm{~cm}$ x $10.0 \mathrm{~mm} \mathrm{I.D.;} 10 \mathrm{~mL} / \mathrm{min}$ Hexanes/iPrOH, 95/5; first eluting enantiomer $(M) \alpha_{\mathrm{D}}^{23}-53$, 99/1 er (c $6.3 \mathrm{mg} / \mathrm{mL}, \mathrm{CHCl}_{3}$ ); second eluting enantiomer $(P) \alpha_{\mathrm{D}}^{23} 56,98.5 / 1.5$ er $\left(c 6.3 \mathrm{mg} / \mathrm{mL}, \mathrm{CHCl}_{3}\right)$. 
<smiles>C=CC1(C)C(=O)N(C)c2c(C)cc(C)cc21</smiles>

\section{1,3,5,7-Tetramethyl-1-vinyl-1,3-dihydroindol-2-one (5a):}

To a dry flask equipped with a stir bar was charged $\operatorname{Pd}_{2}(\mathrm{dba})_{3}(9.2 \mathrm{mg}, 10 \mu \mathrm{mol})$ and (tert$\mathrm{Bu}_{3} \mathrm{PH} \cdot \mathrm{BF} 4(11.6 \mathrm{mg}, 40 \mu \mathrm{mol})$. The flask was capped with a septum and backfilled with Ar. A solution of enantioenriched anilide $(34.3 \mathrm{mg}, 100 \mu \mathrm{mol})$ in degassed toluene $(1 \mathrm{~mL})$ was added by syringe, followed by triethyl amine $(33 \mu \mathrm{L}, 14 \mu \mathrm{mol})$. The reaction mixture was stirred at room temperature for $24 \mathrm{~h}$. The reaction mixture was diluted with $\mathrm{CH}_{2} \mathrm{Cl}_{2}(5 \mathrm{~mL})$ and filtered through a pad of celite. The resulting solution was concentrated in vacuo to generate a dark brown crude solid. The crude material was purified by silica gel flash chromatography (hexanes/ethyl ether, 6/4) to give a yellow oil. The atropisomer (-)-(M) (99.5/0.5 er, first eluting enantiomer) yielded a yellow oil $(R)-(+)$ in $85 \%$ yield $(85.5 / 14.5,+37, c 7.3 \mathrm{mg} / \mathrm{mL}$, second eluting enantiomer). The atropisomer $(+)-(P)(98.5 / 1.5$ er, second eluting enantiomer) yielded a yellow oil $(S)-(-)$ in $92 \%$ yield $(86.5 / 13.5 \mathrm{er},-38$, c $8.9 \mathrm{mg} / \mathrm{mL}$, first eluting enantiomer): IR (neat, $\mathrm{KBr}, \mathrm{cm}^{-1}$ ) 2972, 2925, 1711, 1635, 1601, 1481, 1342, 1088; ${ }^{1} \mathrm{H}$ NMR (300 MHz, $\left.\mathrm{CDCl}_{3}\right)$ ठ $1.56(\mathrm{~s}, 3 \mathrm{H}), 2.23(\mathrm{~s}, 3 \mathrm{H}), 2.55(\mathrm{~s}, 3 \mathrm{H}), 3.48(\mathrm{~s}, 3 \mathrm{H}), 5.13(\mathrm{~d}, J=17.0 \mathrm{~Hz}, 1 \mathrm{H}), 5.15(\mathrm{~d}, J=$ $10.6 \mathrm{~Hz}, 1 \mathrm{H}), 5.91(\mathrm{dd}, J=17.0,10.6 \mathrm{~Hz}, 1 \mathrm{H}), 6.83(\mathrm{~s}, 2 \mathrm{H}) ;{ }^{13} \mathrm{C} \mathrm{NMR}(75 \mathrm{MHz}, \mathrm{CDCl} 3) \delta$ $18.9,20.8,22.7,29.6,50.6,115.0,119.5,122.5,131.9,132.2,133.6,138.3,138.5,179.3$; HRMS (EI) calcd for $\mathrm{C}_{14} \mathrm{H}_{17} \mathrm{NO}$ 215.1310, found 215.1308; LRMS (EI) $m / z 215\left(\mathrm{M}^{+}, 100\right), 200$ (58), $173(46)$.<smiles>C/C=C(/C)C(=O)N(C)c1c(C)cc(Br)cc1I</smiles>

$N$-(4-Bromo-2-iodo-6-methyl)- $N$,2-dimethyl-(2E)-butenamide (4b): 
To a round bottom flask equipped with a stir bar, a slurry of $\mathrm{NaH}(95 \%, 110 \mathrm{mg}, 4.56 \mathrm{mmol})$ and THF $(15 \mathrm{~mL})$ was created. The flask was capped, backfilled with argon, and its contents cooled to $0{ }^{\circ} \mathrm{C}$. The amide $(1.5 \mathrm{~g}, 3.8 \mathrm{mmol})$ was dissolved in THF $(20 \mathrm{~mL})$ and added dropwise via syringe to the slurry. After stirring for 15 min methyl iodide (284 $\mu \mathrm{L}, 4.56 \mathrm{mmol})$ was added via syringe. After a further $1 \mathrm{~h}$ water was carefully added to quench remaining hydride. The resulting biphasic mixture was extracted with diethyl ether $(3 \times 25 \mathrm{~mL})$. The combined organic fractions were washed with brine, dried over $\mathrm{MgSO}_{4}$, and concentrated in vacuo. The resulting crude material was purified by silica gel chromatography (hexanes/diethyl ether, 7/3) to give a tan, crystalline solid (mp $139-140{ }^{\circ} \mathrm{C}$ ) in $98 \%$ yield: IR (thin film, $\mathrm{CH}_{2} \mathrm{Cl}_{2}, \mathrm{NaCl}, \mathrm{cm}^{-1}$ ) 2918, 1658, 1622, 1440, 1355; Note: Compound exists as a 2.6:1 mixture of $\mathrm{E} / \mathrm{Z}$ amide rotamers in $\mathrm{CDCl}_{3}$; signals assigned to the major isomer are in bold and those of a mixture italicized. ${ }^{1} \mathrm{H}$ NMR $\left(300 \mathrm{MHz}, \mathrm{CDCl}_{3}\right) \delta \mathbf{1 . 5 0}(\mathrm{dq}, J=6.9,1.1 \mathrm{~Hz}, 3 \mathrm{H}), \mathbf{1 . 6 8}(\mathrm{qn}, J=1.2 \mathrm{~Hz}, 3 \mathrm{H}), 1.78(\mathrm{dq}, J$ = 6.9, $1.1 \mathrm{~Hz}, 3 \mathrm{H}), 1.97(\mathrm{qn}, J=1.2 \mathrm{~Hz}, 3 \mathrm{H}), 2.26(\mathrm{~s}, 3 \mathrm{H}), \mathbf{3 . 1 4}(\mathrm{s}, 3 \mathrm{H}), 3.18(\mathrm{~s}, 3 \mathrm{H}), \mathbf{5 . 7 6}$ (qq, $J=6.9,1.2 \mathrm{~Hz}, 1 \mathrm{H}), 5.94$ (qq, $J=6.9,1.2 \mathrm{~Hz}, 1 \mathrm{H}), 7.36$ (dd, $J=2.2,0.5 \mathrm{~Hz}, 1 \mathrm{H}), 7.39$ $(\mathrm{dd}, J=2.2,0.5 \mathrm{~Hz}, 1 \mathrm{H}), 7.85(\mathrm{dd}, J=2.2,0.5 \mathrm{~Hz}, 1 \mathrm{H}) ;{ }^{13} \mathrm{C} \mathrm{NMR}\left(75 \mathrm{MHz}, \mathrm{CDCl}_{3}\right) \delta 13.6$, 13.9, 19.2, 35.8, 101.3, 121.5, 130.7, 131.9, 134.0, 139.0, 139.7, 144.8, 172.5; HRMS (EI) calcd for $\mathrm{C}_{13} \mathrm{H}_{15} \mathrm{BrINO}$ 406.9382, found 406.9370; LRMS (EI) $\mathrm{m} / z 408$ (M+, 43), 280 (91), 117 (60), 96 (64), 83 (100). The racemate was submitted to preparative chiral HPLC separation (Regis Technologies $(S, S)$-Whelk-O 1; $25 \mathrm{~cm}$ x $10.0 \mathrm{~mm}$ I.D.; $10 \mathrm{~mL} / \mathrm{min}$ Hexanes/PrOH, 9/1; first eluting enantiomer $(M) \alpha_{\mathrm{D}}{ }^{23}-41,99 / 1$ er $\left(c 6.7 \mathrm{mg} / \mathrm{mL}, \mathrm{CHCl}_{3}\right)$; second eluting enantiomer $(P)$ $\alpha_{\mathrm{p}^{23}} 38,99 / 1 \mathrm{er}\left(c 11.6 \mathrm{mg} / \mathrm{mL}, \mathrm{CHCl}_{3}\right)$.

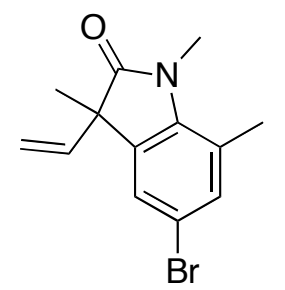

\section{5-Bromo-1,3,7-trimethyl-1-vinyl-1,3-dihydroindol-2-one (5b):}


To a dry flask equipped with a stir bar was charged $\mathrm{Pd}_{2}(\mathrm{dba})_{3}(9.2 \mathrm{mg}, 10 \mu \mathrm{mol})$ and (tert$\mathrm{Bu}_{3} \mathrm{PH} \cdot \mathrm{BF}_{4}(11.6 \mathrm{mg}, 40 \mu \mathrm{mol})$. The flask was capped with a septum and backfilled with Ar. A solution of enantioenriched anilide $(41 \mathrm{mg}, 100 \mu \mathrm{mol})$ in degassed toluene $(1 \mathrm{~mL})$ was added by syringe, followed by triethyl amine ( $33 \mu \mathrm{L}, 24 \mu \mathrm{mol})$. The reaction mixture was stirred at room temperature for $24 \mathrm{~h}$. The reaction mixture was diluted with $\mathrm{CH}_{2} \mathrm{Cl}_{2}(5 \mathrm{~mL})$ and filtered through a pad of celite. The resulting solution was concentrated in vacuo to generate a dark brown crude solid. The crude material was purified by silica gel flash chromatography (hexanes/ethyl ether, $6 / 4)$ to give 159 as a yellow oil. The atropisomer (-)-( $M$ ) (99.5/0.5 er, first eluting enantiomer) yielded a white crystalline solid $(R)-(+)$ in $77 \%$ yield $(89 / 11,+47, c 12.1 \mathrm{mg} / \mathrm{mL}$, second eluting enantiomer). The atropisomer $(+)-(P)(97.5 / 2.5 \mathrm{er}$, second eluting enantiomer) yielded a white crystalline solid (S)-(-) in $69 \%$ yield $(89 / 11 \mathrm{er},-42$, c $10.3 \mathrm{mg} / \mathrm{mL}$, first eluting enantiomer): IR (thin film, $\mathrm{CH}_{2} \mathrm{Cl}_{2}, \mathrm{NaCl}, \mathrm{cm}^{-1}$ ) 2970, 2929, 1713, 1600, 1463; ${ }^{1} \mathrm{H}$ NMR $\left(300 \mathrm{MHz}, \mathrm{CDCl}_{3}\right) \delta$ 1.47 (s, 3 H), 2.57 (s, 3 H), 3.50 (s, 3 H), 5.13 (d, $J=17.2 \mathrm{~Hz}, 1 \mathrm{H}), 5.18$ (d, $J=10.4 \mathrm{~Hz}, 1 \mathrm{H})$, $5.89(\mathrm{dd}, J=17.2,10.4 \mathrm{~Hz}, 1 \mathrm{H}), 7.12(\mathrm{dd}, J=2.1,0.5 \mathrm{~Hz}, 1 \mathrm{H}), 7.17-7.18(\mathrm{~m}, 1 \mathrm{H}) ;{ }^{13} \mathrm{C}$ NMR $\left(75 \mathrm{MHz}, \mathrm{CDCl}_{3}\right) \delta$ 18.8, 22.6, 29.6, 50.7, 114.9, 115.6, 121.8, 124.9, 134.1, 135.3, 137.7, 139.9, 178.8; HRMS (EI) calcd for $\mathrm{C}_{13} \mathrm{H}_{14} \mathrm{BrNO} 279.0259$, found 279.0258; LRMS (EI) $\mathrm{m} / z 279\left(\mathrm{M}^{+}\right.$, 100), 264 (23), 250 (20), 237 (40), 185 (33), 157 (45). 
Figure S1. ORTEP Diagram of $(P)-\mathbf{4 b}$

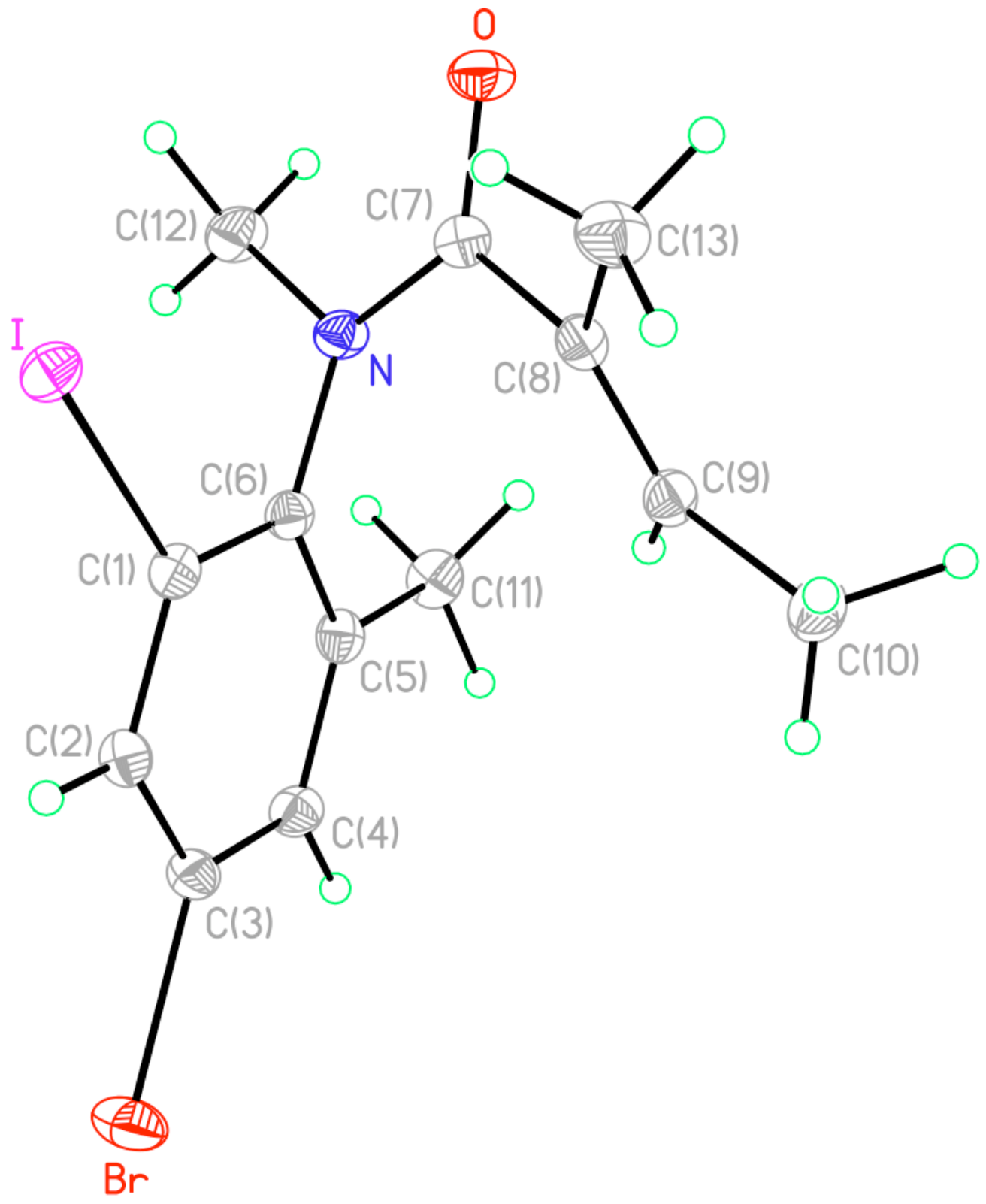


Figure S2. ORTEP Diagram of $(S)-\mathbf{5 b}$

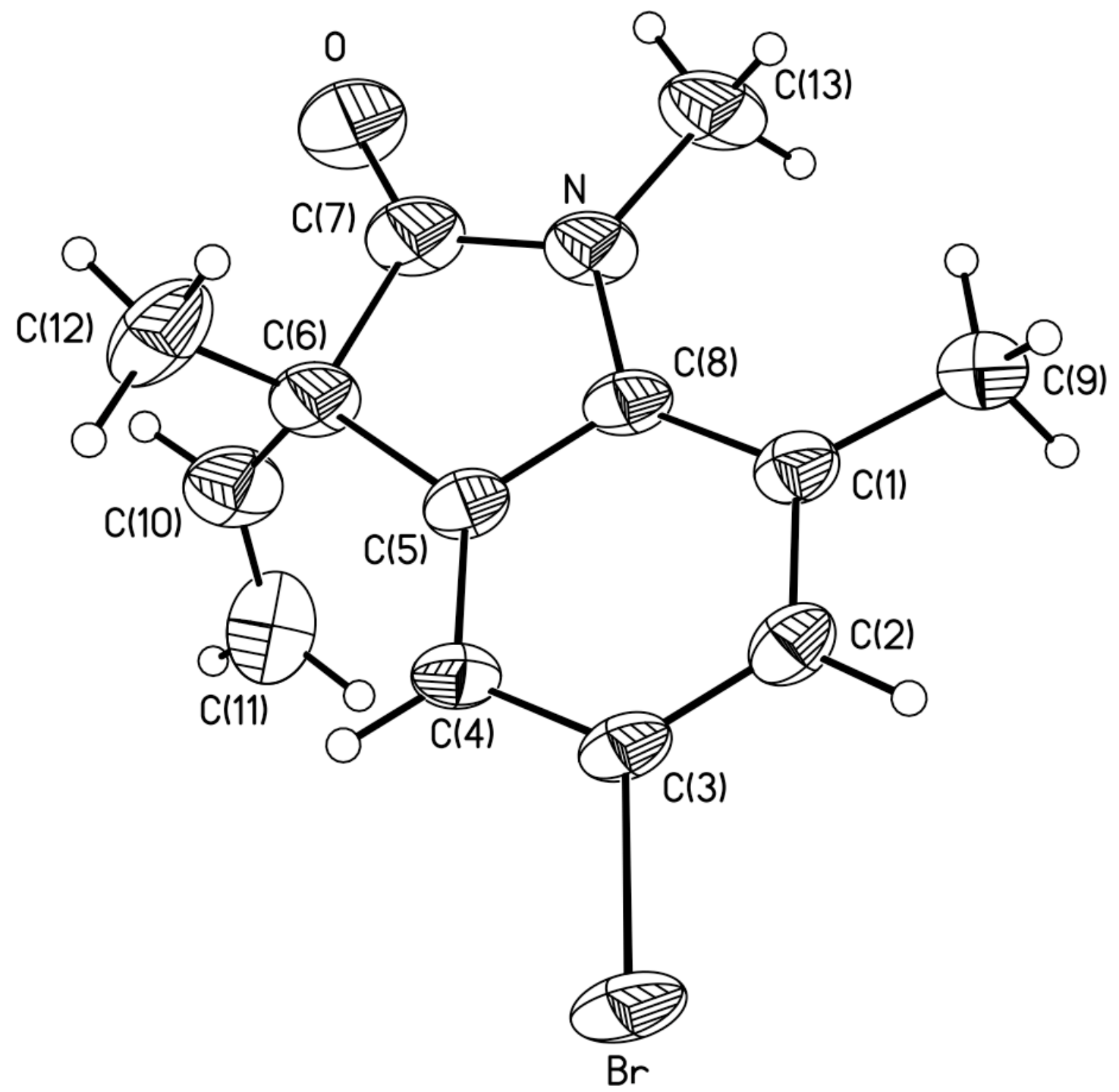




\section{Chiral Chromatography}

Analytical method:

Column: Regis Technologies, Inc. (S,S)-Whelk-01; 25cm x 4.6mm I.D.

Injection volume: $10 \mu \mathrm{L}$

Semi-preparative method:

Column: Regis Technologies, Inc. (S,S)-Whelk-01; 25cm x 10.0mm I.D.

Injection volume: $1 \mathrm{~mL}$

The two enantiomers of $\mathbf{4 a}$ and $\mathbf{4 b}$ were resolvable on a semi-preparative column, eluting with $10 \%$ iso-propanol in hexane (10 $\mathrm{mL} / \mathrm{min}, 25-50 \mathrm{mg}$ per injection). Enantiomerically-enriched $\mathbf{4 b}$ (first eluting enantiomer by HPLC) was dissolved in hexanes $(5 \mu \mathrm{g} / \mathrm{mL})$ and stirred at room temperature $(296 \mathrm{~K})$. At the given intervals of time about $5 \mu \mathrm{L}$ of the solution was injected into the HPLC and the \% ee was measured using peak integrations.

$\begin{array}{cc}4 \mathrm{~b} & \mathrm{~T}=296 \mathrm{~K} \\ \text { Time }(\mathrm{sec}) & \% \text { ee } \\ 0 & 99.0 \\ 16140 & 98.0 \\ 66540 & 93 \\ 107640 & 90 \\ 896940 & 42 \\ 1383000 & 32\end{array}$




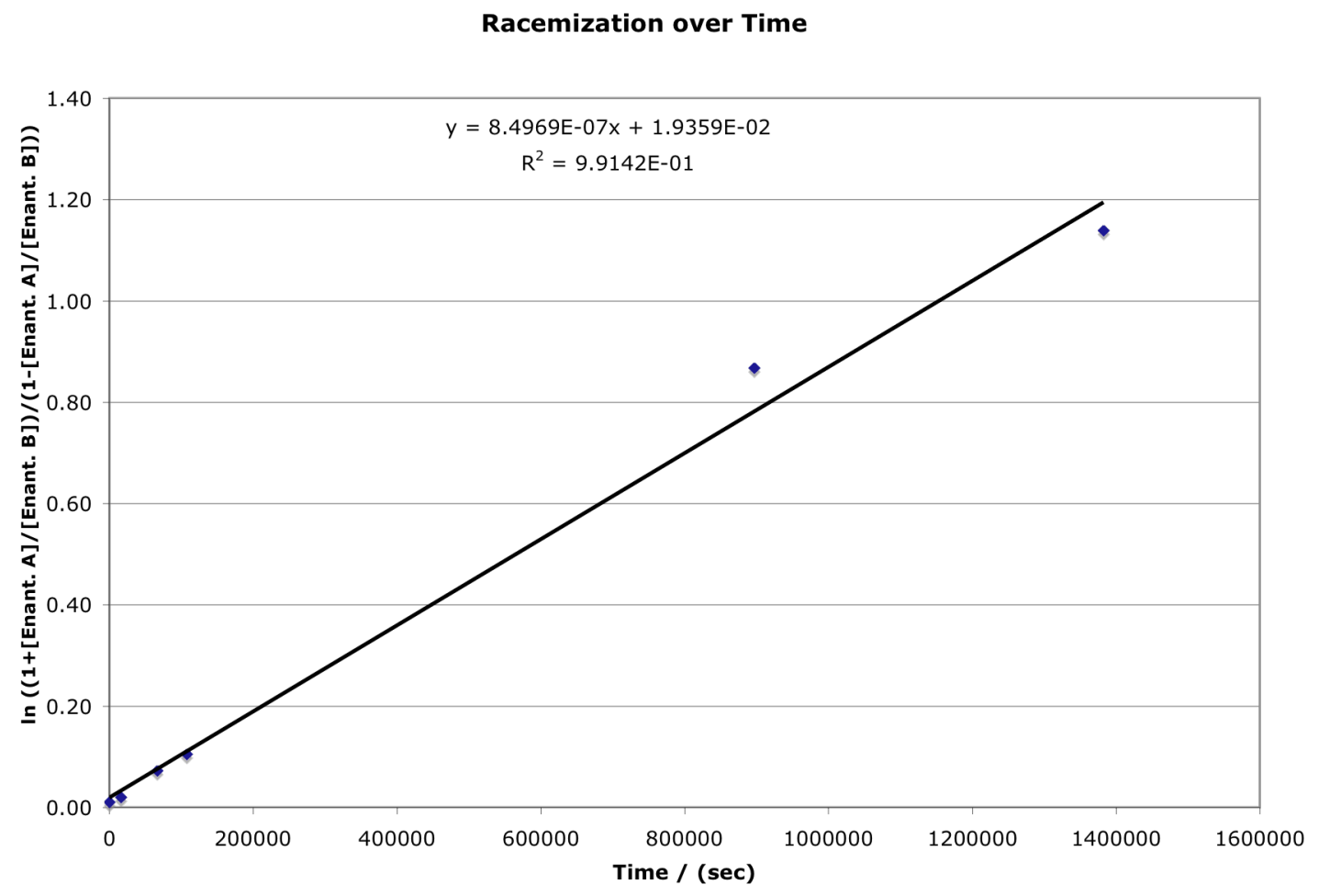

$\ln \left[\frac{1+[\text { Enant. } A] /[\text { Enant. B] }}{1-[\text { Enant. } A] /[\text { Enant. B] }}\right]=2 \mathrm{kt}+\mathrm{C}$

Plot $\quad \ln \left[\begin{array}{c}1+[\text { Enant. A]/[Enant. B] } \\ 1-[\text { Enant. A]/[Enant. B] }\end{array}\right]$ vs Time (seconds)

where

-slope $=2 \mathrm{k}$

$-C$ is equal to zero if starting material se is $\% 100$

-Enant. A represents the most prominent enantiomer in

solution during the experiment (Enant. $B$ the least)

$k=1 / 2$ slope $=1 / 2 *(8.50 \mathrm{E}-07)=4.25 \mathrm{E}-07 \mathrm{~s}^{-1}$

$\mathrm{K}^{\ddagger}=\mathrm{kh} / \mathrm{kT}=\frac{\left(4.25 \mathrm{E}-07 \mathrm{~s}^{-1}\right)(6.626 \mathrm{E}-34 \mathrm{Js})}{(1.381 \mathrm{E}-23 \mathrm{~J} / \mathrm{K})(296 \mathrm{~K})}$

$\mathrm{K}^{\ddagger}=6.89 \mathrm{E}^{-20}$ and $\Delta \mathrm{G}^{\ddagger}=-\mathrm{RT} \operatorname{lnK}$

$\Delta \mathrm{G}_{\text {rot }}^{\ddagger}=1.08 \times 10^{5} \mathrm{~J} / \mathrm{mol}$ or $26.0 \mathrm{kcal} / \mathrm{mol}$ 